\title{
COMPARISON OF COOPERATIVE DIVERSITY PROTOCOLS IN VARIOUS RELAY LOCATIONS THROUGH NETWORK CODING

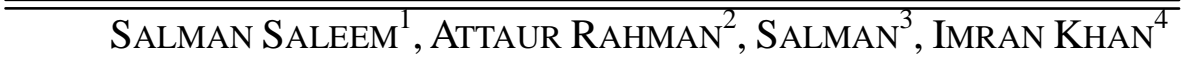 \\ ${ }^{1,2,4}$ Department of Telecommunication Engineering University of Engineering \& Technology Peshawar \\ Khyber Pakhtunkhwa, Pakistan \\ ${ }^{3}$ Department of Computer Sciences, Abdul Wali Khan University Mardan, Pakistan \\ 1engrsalmansaleem@gmail.com; ${ }^{2}$ engr.attaurrahman12@gmail.com \\ ${ }^{3}$ salman@awkum.edu.pk; ${ }^{4}$ imrankhan@uetpeshawar.edu.pk
}

\begin{abstract}
Revised June 2015
ABSTRACT. In this paper, the performance of Network Coded (NC) based cooperative network for various relay location over Rayleigh fading channels is studied. Comparisons of Amplify and Forward (AAF), Detect and Forward (DTF) and Decode and forward $(D C F)$ protocols for the proposed system are shown. The performance of relays in AAF, DTF and DCF is analyzed in terms of bit error rate $(B E R)$ vs signal to noise ratio (SNR). Matlab is used to build Monte-Carlo link level simulation. The effect of relays at different position is studied.

Keywords: Network Coding; Cooperative Communication; Maximum Ratio Combining; Spatial Diversity; Bit error rate.
\end{abstract}

1. Introduction. Cooperative Communication enables single antenna to communicate in multi user environment to generate a multiple antenna transmitter to achieve transmit diversity [1]. Installing more than one antenna on single wireless device creates a lot of difficulties. In Cooperative communication, the information from source is carried to destination with the help of neighboring nodes present in between them. These nodes made copies of the message and deliver to the destination in improved form. The cooperative diversity concept is given in [2-4]. Amplify and Forward AAF, Decode and Forward DCF and Detect and Forward DTF are the main protocols used in Cooperative Communication [5], [6]. In AAF the information is amplified 1st then it is forwarded to destination. In DCF the message is decoded then it is sent to destination. While in DTF, the message is sent to destination after demodulation and modulation strategies. Relays play an important role in long Term Evolution (LTE) advanced standardization for increment in coverage area [7]. Network Coding can be used to create diversity and enhance network throughput. The main idea about Network Coding (NC) was for the first time given in [8] by Ahlswede et al for wired networks which further implemented on wireless networks and showed a promising results in the performance. Signals received at the relays are the superposition of transmitted signals from source, the common AAF scheme is type of NC at signal level [9, 10]. In [11], [12] digital network coding has been compared with Analog Network Coding (ANC) at packet level for duplex communication between sources. The ANC for two way communication for time asynchronous is shown in [13]. Every single relay by using Toeplitz matrix, converts the received asynchronous signal in the 1st time slot and broadcast them to the terminals in second time slot. The authors in [14] divided the ANC protocol into Multiple Access Channel (MAC) phase and Broadcast Channel (BC) phase. In MAC phase the received signal is amplified by relay, while in $\mathrm{BC}$ phase the amplified signal is broadcast to the terminals. The performance of an outage optimal bi-directional Two-Way Opportunistic Relaying (TWOR) with ANC protocol over independent non identical distributed Nakagami-m fading channels is explained in [15]. The optimum power allocation scheme for ANC protocol is proposed in [16], which reduces the outage probability and increases the total number of mutual information of ANC protocol. In [17] space time analog network protocol (STANC) is given. On the basis of moment generation function 
approach, the performance of STANC is studied in terms of SER.

In this paper, we have analyzed different protocols i.e., AAF, DTF and DCF for NC based relay networks in Rayleigh fading channels. Moreover, the distance effect of relays on system at various location is studied. The BER analysis results for different protocols are compared with respect to relays location from the source. It is concluded that changing the location of relays have great effect on the performance of AAF, DTF and DTF in terms of BER.

The rest of the correspondence is organized as follows: Section II describes the system model for the wireless relay channel, results of our system model are suggested in Section III. Finally, Section IV provides the conclusions and future work.

2. System Model. We consider a wireless network in figure 1, consisting of single source $\boldsymbol{S}$, one destination $\boldsymbol{D}$ and two relays represented by $\boldsymbol{R}_{\boldsymbol{I}}$ and $\boldsymbol{R}_{2}$. Every terminal is equipped with single antenna. We are considering MRC technique at the destination to combine signals of direct link and indirect link. The orthogonal channels are used for transmission discussed in [6]. The transferring information is modulated with Binary Phase Shift Keying (BPSK). We consider a Rayleigh flat fading channel with Additive White Gaussian Noise (AWGN) for links between source, relays and destination.

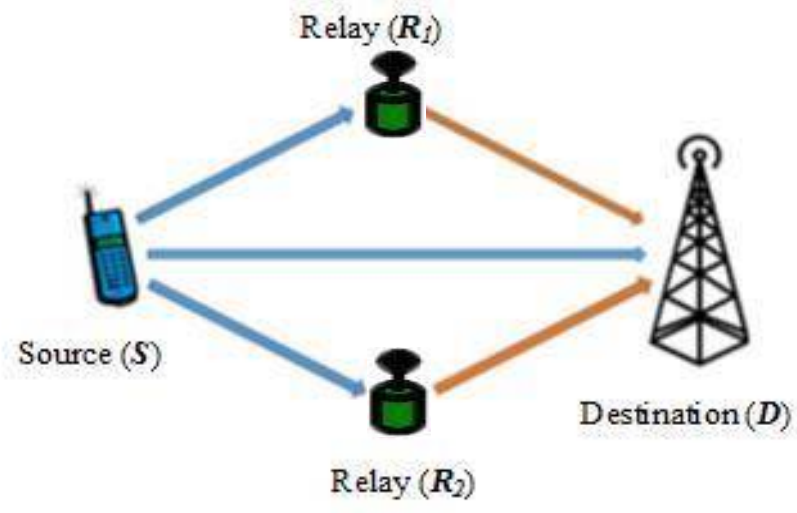

Figure 1. Cooperative Network with single source (S), two relays $\left(\mathrm{R}_{1}\right.$ and $\left.\mathrm{R}_{2}\right)$ and a destination (D)

Consider a signal $a_{\bar{i}}$ is transmitted from the source, the general equation for signal received $b_{i}$ can be written as:

$$
b_{i}=h_{i} a_{i}+n o_{i}
$$

Whereas, $h_{i} a_{i}$ Shows the attenuated signal and $n o_{i}$ represents the noise.

The two relays are located at different locations represented by $d_{11}, d_{12}, d_{21}$ and $d_{22}$ between $S$ and $\boldsymbol{D}$ whose distance can be formulated as:

$$
\begin{aligned}
& d_{11}=\sqrt{d_{1}^{2}+0.25^{2}} \\
& d_{12}=\sqrt{\left(1-d_{1}\right)^{2}+0.25^{2}} \\
& d_{21}=\sqrt{d_{2}^{2}+0.25^{2}} \\
& d_{22}=\sqrt{\left(1-d_{2}\right)^{2}+0.25^{2}}
\end{aligned}
$$


The complete transmission is divided into two time slots i.e., time slot I and time slot II. In first slot shown in figure 2, the source $\boldsymbol{S}$ transmits signals to both relays $\boldsymbol{R}_{\boldsymbol{1}}$ and $\boldsymbol{R}_{2}$ as well as to destination $\boldsymbol{D}$. ${ }^{b_{R_{1}},} b_{R_{2}}$ and $b_{D}$ represent signal received by $\boldsymbol{R}_{1}, \boldsymbol{R}_{2}$ and $\boldsymbol{D}$ from $S$.

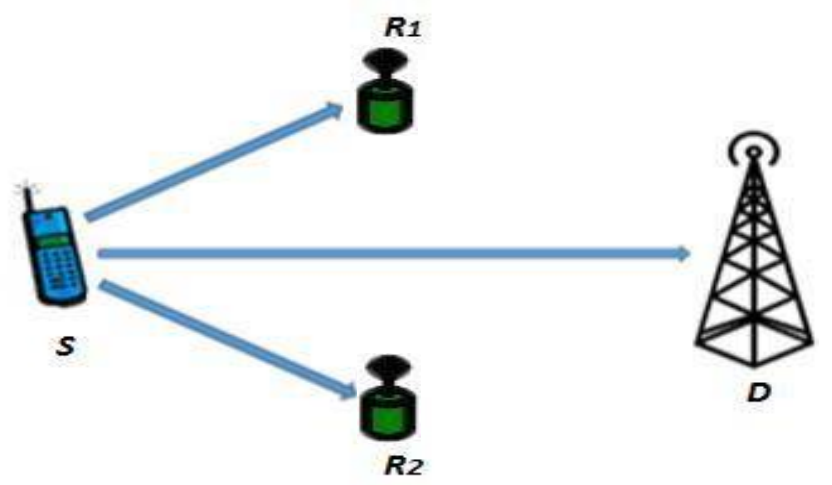

Figure 2. Time Slot I $\left(S \rightarrow R_{1}, R_{2}, D\right)$

The signals transmitted during this stage from [18], [19] are given as:

$$
\begin{aligned}
& b_{R_{1}}=\left(d_{k}\right)^{-u} \sqrt{E_{S R 1}} h_{R 1} a_{1}+n o_{R 1} \\
& b_{R_{2}}=\left(d_{k}\right)^{-u} \sqrt{E_{S R 2}} h_{R 2} a_{1}+n o_{R 2} \\
& b_{D}=\left(d_{k}\right)^{-u} \sqrt{E_{S D}} h_{S D} a_{1}+n o_{D}
\end{aligned}
$$

Where $E_{S R 1}, E_{S R 2}$ and $E_{S D}$ are the average energies received at $\boldsymbol{R}_{1}, \boldsymbol{R}_{2}$ and $\boldsymbol{D}$ respectively and $d_{k}$ shows its respective distance. $-\boldsymbol{u}$ represents path loss exponent. $h_{R 1} a_{1}, h_{R 2} a_{1}, h_{S D} a_{1}, n o_{R 1}, n o_{R 2}$, no $o_{D}$ represents attenuated signals and additive white Gaussian noise (AWGN) between $S$ and $\boldsymbol{R}_{\boldsymbol{l}}, \boldsymbol{S}$ and $\boldsymbol{R}_{\mathbf{2}}$ and $\boldsymbol{S}$ and $\boldsymbol{D}$ links. While, the second time slot shown in figure 3 , relays transmit data to destination by using either AAF, DCF or DTF protocol.

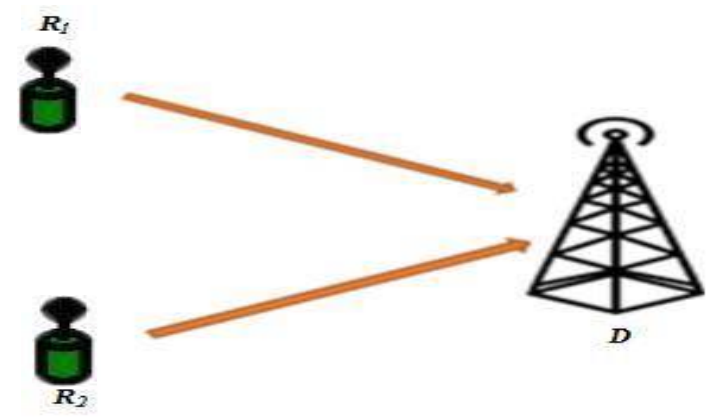

Figure 3. Time Slot II $\left(\mathrm{R}_{1}, \mathrm{R}_{2} \rightarrow \mathrm{D}\right)$

2.1. Amplify and Forward (AAF). AAF protocol is mostly used in that condition when relay has short amount of time for encoding and decoding. In this case, the relays first amplify the signals (by adding amplification factor) and then broadcast to the destination. The drawback of this protocol is during amplification, the noise is also amplified.

The amplified signals $a_{a m p 1}$ and $a_{a m p 2}$ from $\boldsymbol{R}_{1}$ and $\boldsymbol{R}_{2}$ respectively to $\boldsymbol{D}$ can be given as: 


$$
\begin{aligned}
& a_{a m p 1}=b_{R 1} * \beta_{k} \\
& a_{a m p 2}=b_{R 2} * \beta_{k}
\end{aligned}
$$

$\beta_{k}$ is the amplification factor and is given by:

$$
\beta_{k}=\sqrt{\frac{1}{\left|h_{R_{1}}\right|^{2}+\left|h_{R 2}\right|^{2}+n c}}
$$

Here the equations for the signals from $\boldsymbol{R}_{I}$ and $\boldsymbol{R}_{2}$ to $\boldsymbol{D}$ will be:

$$
\begin{aligned}
& b_{a f R 1}=\left(d_{k}\right)^{-u} \sqrt{E_{R 1 S}} a_{a m p 1} \cdot h_{R 1}+n o_{R 1} \\
& b_{a f R 2}=\left(d_{k}\right)^{-u} \sqrt{E_{R 2 S}} a_{a m p 2} \cdot h_{R 2}+n o_{R 2}
\end{aligned}
$$

2.2. Decode and Forward (DCF). Generally in wireless communication transmission, we use digital modulation. In that respect, Decode and forward protocol is most preferable option for wireless communication. Here the signals are first decoded and then forwarded to destination so that no noise is amplified in a broadcast signals. The signals from $\boldsymbol{R}_{\boldsymbol{I}}$ and $\boldsymbol{R}_{\mathbf{2}}$ to $\boldsymbol{D}$ in this case can be represented by:

$$
\begin{aligned}
& b_{d c f R 1}=\left(d_{k}\right)^{-u} \sqrt{E_{R 1 s}} a_{d c f 1} \cdot h_{R 1}+n o_{R 1} \\
& b_{d c f R 2}=\left(d_{k}\right)^{-u} \sqrt{E_{R 2 s}} a_{d c f 2} \cdot h_{R 2}+n o_{R 2}
\end{aligned}
$$

Where ${ }^{a_{d c f 1} \text { and }}{ }^{a_{d c f 2}}$ are the BPSK signals after re-encoding.

2.3. Detect and Forward (DTF). In this protocol both relays first demodulated the received signals, then modulate and finally send the binary signals to destination.

Equalizing at relays:

$$
\begin{aligned}
& e q_{R 1}=b_{R 1} \times z^{*} h_{R 1} \\
& e q_{R 2}=b_{R 2} \times z^{*} h_{R 2}
\end{aligned}
$$

Where, $z^{*}$ shows the conjugate of $h_{R 1}$ and $h_{R 2}$

$$
\begin{aligned}
& d e c_{d t f_{R 1}}=\operatorname{sign}\left(\mathbb{R} \times e q_{\mathrm{R} 1}\right) \\
& d e c_{d t f_{R 2}}=\operatorname{sign}\left(\mathbb{R} \times e q_{\mathrm{R} 2}\right)
\end{aligned}
$$

The final equations for this stage from $\boldsymbol{R}_{\boldsymbol{I}}$ and $\boldsymbol{R}_{2}$ to $\boldsymbol{D}$ are:

$$
\begin{aligned}
& b_{d t f R 1}=\left(d_{k}\right)^{-u} \sqrt{E_{s r 2}} a_{d t f 1} \cdot h_{R 1}+n o_{R 1} \\
& b_{d t f R 2}=\left(d_{k}\right)^{-u} \sqrt{E_{s r 2}} a_{d t f 2} \cdot h_{R 2}+n o_{R 2}
\end{aligned}
$$

2.4. MRC. The destination receives the several signals and combines them using Maximum Ratio Combining (MRC).

$$
b_{d}[n]=\sum_{i=1}^{k} h_{i u d}[n] . b_{i u d}[n]
$$


The normalizing factors $w_{1}$ and $w_{2}$ from [18], [19] can be expressed as:

$$
\begin{aligned}
& w_{1}=\sqrt{\frac{E_{R_{1} D}}{\left(E_{S R_{1}}+N_{0}\right)\left[\left.h_{R_{1} D}\right|^{2}+1\right.}} \\
& w_{2}=\sqrt{\frac{E_{R n D}}{\left(E_{S R 2}+N_{0}\right)\left[\left.h_{R 2 D}\right|^{2}+1\right.}}
\end{aligned}
$$

Let, $w_{1} \cong w_{2} \cong w$, then the signals received by $\boldsymbol{D}$ from second slot can be expressed as:

$$
\begin{aligned}
& b_{2}=\left(\frac{1}{w}\left(\left(d_{S R 1}\right)^{-u}\left(d_{R 1 D}\right)^{-u} \sqrt{\frac{E_{S R 1} E_{R 1 D}}{E_{S R 1}+n_{o}}} h_{S R 1} h_{R 1 D}\right)\right) a_{1} \\
& +\left(\left(d_{S R 2}\right)^{-u}\left(d_{R 2 D}\right)^{-u} \sqrt{\frac{E_{S R 2} E_{R n D}}{E_{S R 2}+n_{0}}} h_{S R 2} h_{R 2 D}\right) a_{1}+\left(d_{S D}\right)^{-u} \frac{1}{w} \sqrt{E_{S D}} h_{S D} a_{2}+n o_{D}
\end{aligned}
$$

Figure 4, shows the positions of relays $\boldsymbol{R}_{\boldsymbol{I}}$ and $\boldsymbol{R}_{2}$ from the $\boldsymbol{S}$

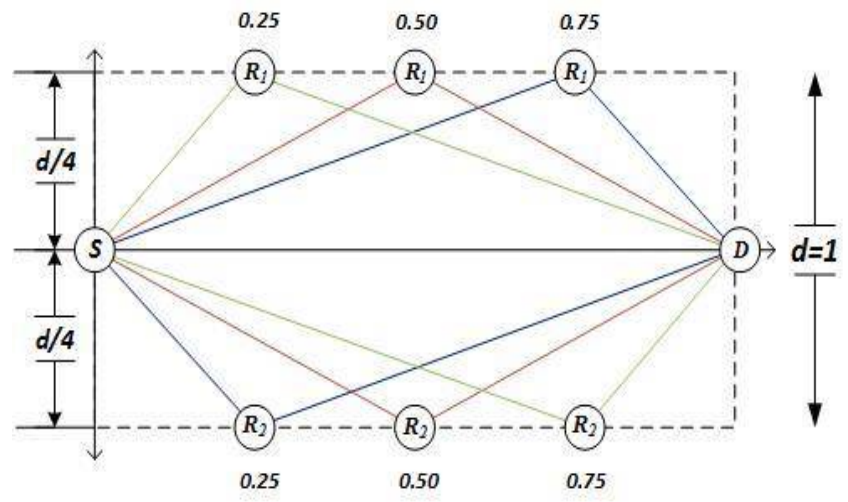

Figure 4. Relays Displacement Optimization

Let $A=\sqrt{E_{S D}} h_{S D}$

$$
B=\frac{1}{w}\left(\left(\sqrt{\frac{E_{S R 1} E_{R 1 D}}{E_{S R 1}+n_{o}}} h_{S R 1} h_{R 1 D}\right)+\left(\sqrt{\frac{E_{S R 2} E_{R 2 D}}{E_{S R 2}+n_{o}}} h_{S R 1} h_{R 1 D}\right)\right)
$$

The derived input - output equation can be given by:

$$
\begin{aligned}
& Y=H X+N \\
& Y^{T}=\left[\begin{array}{ll}
y_{1} & y_{2}
\end{array}\right] \\
& X^{T}=\left[\begin{array}{ll}
x_{1} & x_{2}
\end{array}\right] \\
& N^{T}=\left[\begin{array}{ll}
n_{1} & n_{2}
\end{array}\right]
\end{aligned}
$$


It is to be noted that this formal model can be applied to any particular interlocking after a further analysis. This is because we have modeled the system and defined the properties based on the requirements of like a real system.

2. Simulation Results. In this section, we will present some simulation results using MATLAB tool to demonstrate the performance of different protocols. We plot the performance curves in terms of an average BER versus SNR [dB]. The simulation results are given for a BPSK modulation over Rayleigh fading channels. We assumed that the source $\boldsymbol{S}$ and both relays $\boldsymbol{R}_{\boldsymbol{I}}$ and $\boldsymbol{R}_{2}$ have same noise variance $N_{o}$. The SNR $\gamma_{\text {can be then calculated as: }}$

$$
\gamma=a^{2} E_{b} / N_{0}
$$

For simplification, the distance between source and destination is normalized to 1 . The approach given in [18] explains two time slot communication.

Figure 5, illustrates the difference between relay assisted transmission with direct transmission (without using relays). The simulation result verifies that communication using relay shows a substantial improvement as compare to direct communication in terms of less BER.

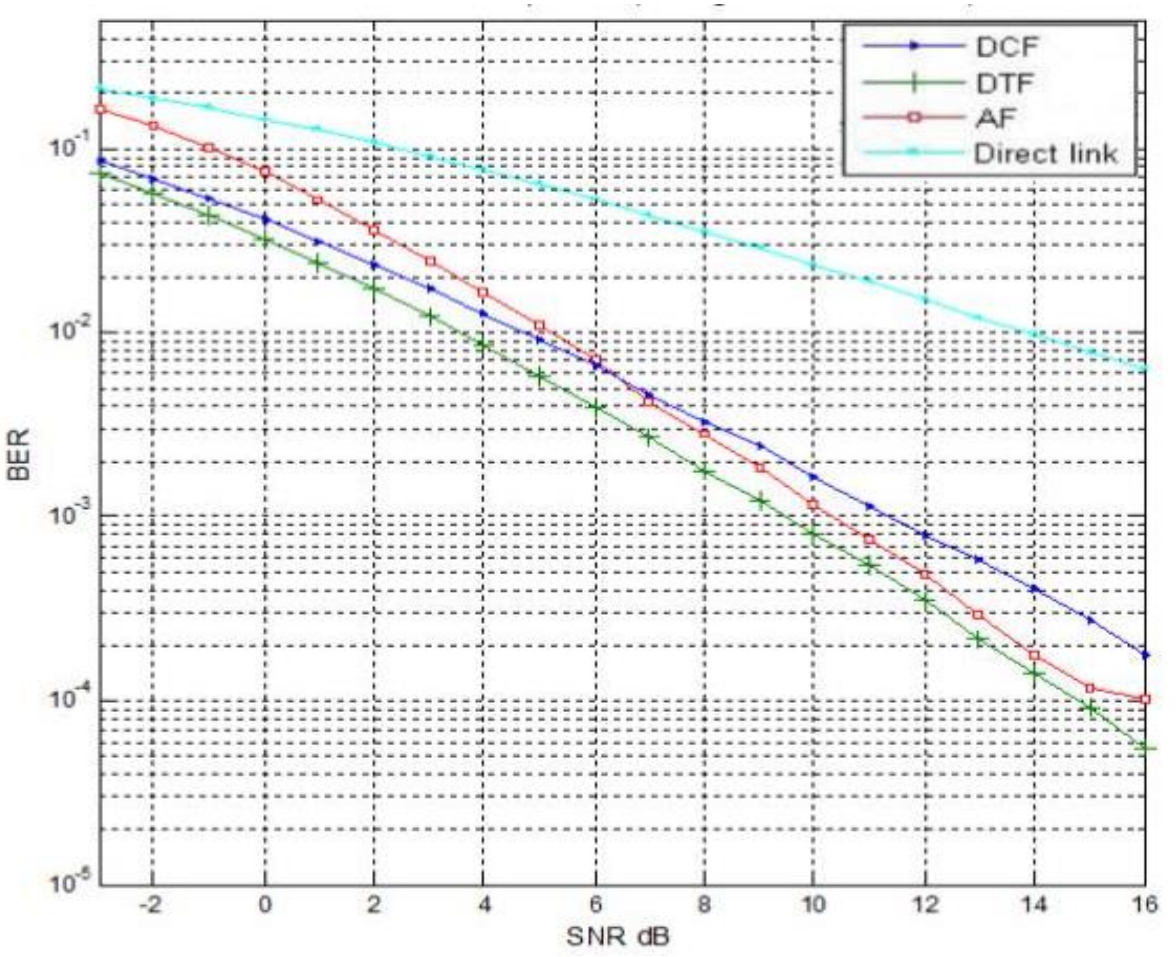

Figure 5. Comparison of Direct Communication with Relay Assisted Communication

In figure 6, we show the average BER performance of AAF, DCF and DTF taking relay R1 at distance 0.5 and relay R2 at distance of 0.25 from the source it is shown in figure that for BER at $10^{-3}$, we get SNR values at $9.8 \mathrm{~dB}, 10.4 \mathrm{~dB}$ and $10.8 \mathrm{~dB}$ for AAF, DCF and DTF respectively. It is clear from this simulation that BER keeps on decreasing for AAF as compared to DCF and DTF at 0.5 and 0.25 . It can be observed that AAF protocol gives better results than DCF and DTF when relays are located at above stated distance.

In Figure 7, we examine the performance of three schemes at 0.75 and 0.25 relays distance from source. It is easy to observe that at BER $10^{-3}$, now DCF protocol shows promising results than DAF, AAF protocols. In this case AAF, DTF and DCF have 13.8 dB SNR, $11.2 \mathrm{~dB}$ SNR and $8 \mathrm{~dB}$ SNR respectively for $10^{-3} \mathrm{BER}$ 


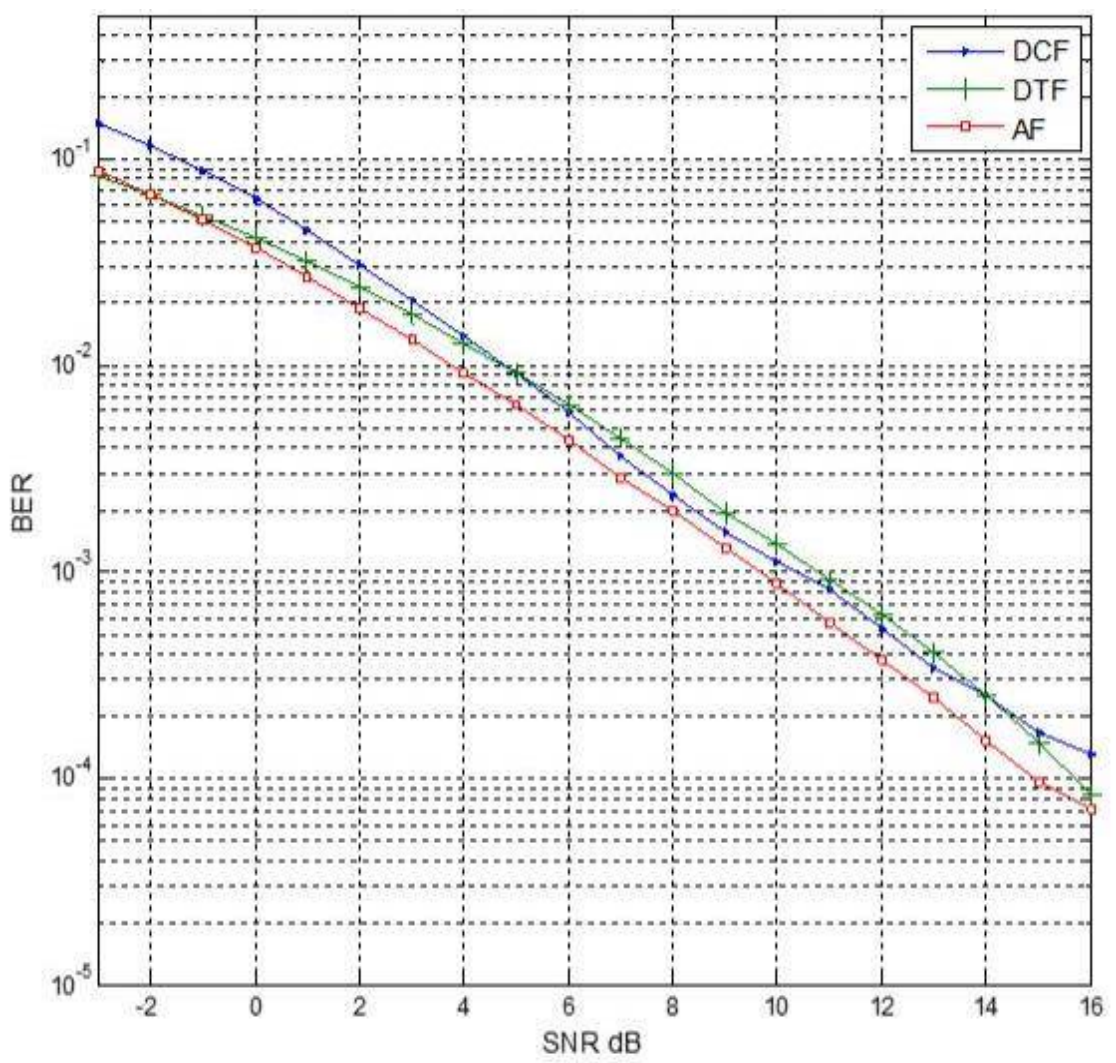

Figure 6. Comparison of AF, DTF and DCF at $\mathrm{R}_{1=} 0.5$ and $\mathrm{R}_{2}=0.25$ distance from $\mathrm{S}$

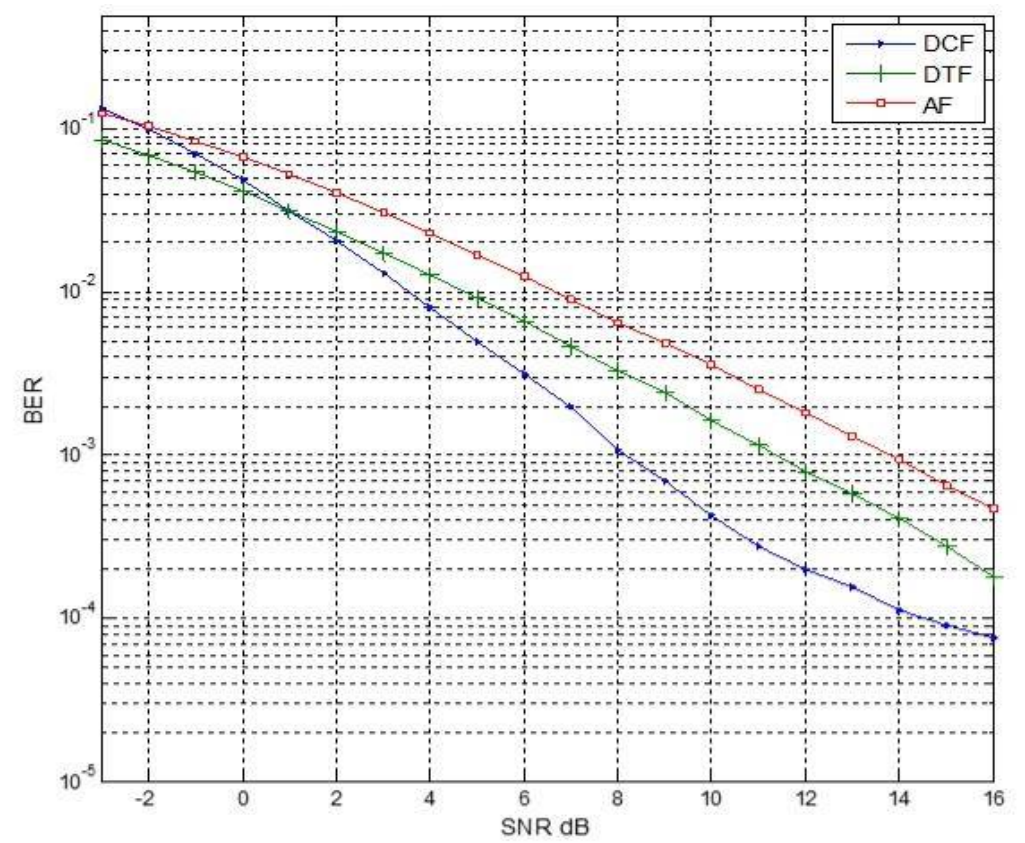

Figure 7. Comparison of $A F, D T F$ and $D C F$ at $R_{1=} 0.75$ and $R_{2}=0.25$ distance from $S$

Finally, in figure 8 we study the average BER comparison between AAF, DTF and DCF when relays position changes to 0.25 and 0.25 from source. Keeping BER at $10^{-3}$, AAF outperforms DCF and DTF protocols in terms of lower BER. The SNR values for AAF, DCF and DTF (for BER $10^{-3}$ ) are $7 \mathrm{~dB}, 10.2 \mathrm{~dB}$ and $11 \mathrm{~dB}$ 
This comparison shows that AAF provides maximum SNR improvement with minimum BER than DCF and DTF protocol at 0.5 and 0.75 distance. Infact, DCF performs better than DTF.

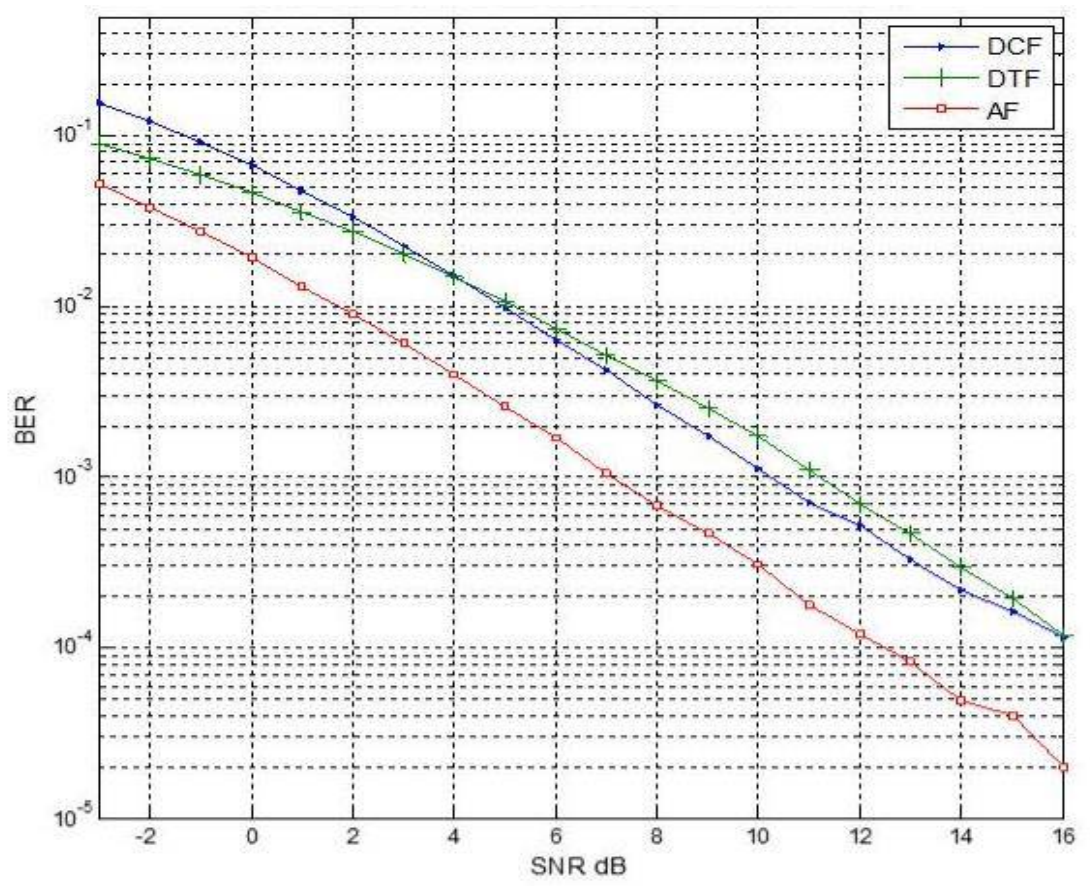

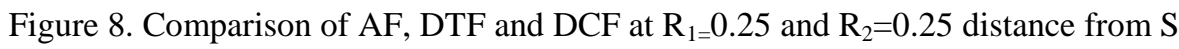

3. Conclusion. Amplify and Forward, Decode and Forward and Detect and Forward protocols in Network Coding on the basis of relays position from the source have been studied. MRC strategy is used to combine all the received signals at destination to achieve spatial diversity. BER vs SNR values are analyzed for AAF, DTF and DTF protocols on the basis of various locations of two relays from the source. It is concluded that relay location has a phenomenal effect on the performance of cooperative communication. Additionally, the performance of communication in $\mathrm{NC}$ can be further improved by using other combining techniques at destination and this work can also be applied on various fading channels for future consideration.

\section{REFERENCES}

[1] Sendonaris, A., Erkip, E., \& Aazhang, B. (2003). User cooperation diversity. Part II. Implementation aspects and performance analysis. Communications, IEEE Transactions on, 51(11), 1939-1948.

[2] Laneman, J. N., Tse, D. N., \& Wornell, G. W. (2004). Cooperative diversity in wireless networks: Efficient protocols and outage behavior. Information Theory, IEEE Transactions on, 50(12), 3062-3080.

[3] Laneman, J. N., \& Wornell, G. W. (2000). Energy-efficient antenna sharing and relaying for wireless networks. In Wireless Communications and Networking Confernce, 2000. WCNC. 2000 IEEE (Vol. 1, pp. 7-12). IEEE.

[4] Nosratinia, A., Hunter, T. E., \& Hedayat, A. (2004). Cooperative communication in wireless networks. Communications Magazine, IEEE, 42(10), 74-80.2004

[5] Chen, D., \& Laneman, J. N. (2006). Modulation and demodulation for cooperative diversity in wireless systems. Wireless Communications, IEEE Transactions on, 5(7), 1785-1794. 
[6] Laneman, J. N., \& Wornell, G. W. (2002, November). Distributed space-time coded protocols for exploiting cooperative diversity in wireless networks. InGlobal Telecommunications Conference, 2002. GLOBECOM'02. IEEE (Vol. 1, pp. 77-81). IEEE.

[7] Mogensen, P. E., Koivisto, T., Pedersen, K. I., Kovacs, I. Z., Raaf, B., Pajukoski, K., \& Rinne, M. J. (2009, May). LTE-advanced: the path towards gigabit/s in wireless mobile communications. In Wireless Communication, Vehicular Technology, Information Theory and Aerospace \& Electronic Systems Technology, 2009. Wireless VITAE 2009. 1st International Conference on (pp. 147-151). IEEE.

[8] Ahlswede, R., Cai, N., Li, S. Y., \& Yeung, R. W. (2000). Network information flow. Information Theory, IEEE Transactions on, 46(4), 1204-1216.

[9] Katti, S., Gollakota, S., \& Katabi, D. (2007, August). Embracing wireless interference: analog network coding. In ACM SIGCOMM Computer Communication Review (Vol. 37, No. 4, pp. 397-408). ACM.

[10] Zhang, S., Liew, S. C., \& Lam, P. P. (2006, September). Hot topic: physical-layer network coding. In Proceedings of the 12th annual international conference on Mobile computing and networking (pp. 358-365). ACM.

[11] Popovski, P., \& Yomo, H. (2007). Wireless network coding by amplify-and-forward for bi-directional traffic flows. Communications Letters, IEEE, 11(1), 16-18.

[12] Chen, M., \& Yener, A. (2008, May). Multiuser two-way relaying for interference limited systems. In Communications, 2008. ICC'08. IEEE International Conference on (pp. 3883-3887). IEEE.

[13] Wang, H. M., Xia, X. G., \& Yin, Q. (2010). A linear analog network coding for asynchronous two-way relay networks. Wireless Communications, IEEE Transactions on, 9(12), 3630-3637.

[14] Zhang, R., Liang, Y. C., Chai, C. C., \& Cui, S. (2009). Optimal beamforming for two-way multi-antenna relay channel with analogue network coding. Selected Areas in Communications, IEEE Journal on, 27(5), 699-712.

[15] Upadhyay, P. K., \& Prakriya, S. (2011). Performance of Two-way opportunistic relaying with analog network coding over Nakagami-fading. Vehicular Technology, IEEE Transactions on, 60(4), 1965-1971.

[16] Yi, Z., Ju, M., \& Kim, I. M. (2011). Outage probability and optimum power allocation for analog network coding. Wireless Communications, IEEE Transactions on, 10(2), 407-412.

[17] Tanoli, S. A. K., Khan, I., Rajatheva, N., \& Adachi, F. (2010). Advances in relay networks: performance and capacity analysis of space-time analog network coding. EURASIP Journal on Wireless Communications and Networking, 2010, 103.

[18] Nabar, R. U., Bolcskei, H., \& Kneubuhler, F. W. (2004). Fading relay channels: performance limits and space-time signal design. Selected Areas in Communications, IEEE Journal on, 22(6), 1099-1109.

[19] Atapattu, S., Rajatheva, N., \& Tellambura, C. (2010). Performance analysis of TDMA relay protocols over Nakagami-fading. Vehicular Technology, IEEE Transactions on, 59(1), 93-104. 